\title{
THE INFRARED BRIGHTNESS OF THE ECLIPTIC PLANE
}

\author{
William T. Reach and Carl Heiles \\ Astronomy Department \\ University of California \\ Berkeley, CA 94720 USA
}

\section{INTRODUCTION}

Prerequisite to the determination of the Galactic and Extragalactic contributions to the infrared background is an accurate removal of the zodiacal emission. The all-sky observations by IRAS in 1983 were made with a variety of observing conditions and with higher sensitivity and angular resolution than previous infrared and optical observations of the zodiacal background. These facts alone mandate a better understanding of the optical properties and spatial distribution of interplanetary dust.

Models of the zodiacal emission (ZE) are typically parameterized density distributions which are integrated over the line of sight appropriate to the particular observation (Hauser 1988; Reach and Heiles 1988). Such models are necessarily simpler than theoretical expectations of the distribution of interplanetary dust. Our goal in this project is to use empirical fits of the variation of the infrared brightness with various observing coordinates in order to determine the distribution of interplanetary dust-without introducing a model. In this contribution we describe our analysis of the brightness of the ecliptic plane.

\section{BRIGHTNESS OF THE ECLIPTIC PLANE}

The variation of the ecliptic plane $\mathrm{ZE}$ brightness was assumed to be separable into a product of several terms, each of which represents the variation with a different coordinate system. We adopted a scheme in which each term separately fit to the ecliptic plane brightness, and the term which produced the largest improvement in $\chi^{2}$ was retained in subsequent fits. In order of importance, the coordinates are listed in Table 1 and discussed individually below.

TABLE 1-Variations of the Infrared Brightness ${ }^{a}$

\begin{tabular}{lcccc}
\hline \hline & $12 \mu \mathrm{m}$ & $25 \mu \mathrm{m}$ & $60 \mu \mathrm{m}$ & $100 \mu \mathrm{m}$ \\
\hline solar elongation & 26. & 39. & 11. & 5. \\
observation date & 1.0 & 1.6 & 0.7 & $?$ \\
geocentric azimuth & 0.5 & 1.0 & 0.2 & $?$ \\
Galactic H I $^{b}$ & 0.067 & 0.038 & 0.241 & 1.02 \\
\hline
\end{tabular}

${ }^{a}$ Amplitude of the variation, in $\mathrm{MJy} \mathrm{Str}^{-1}$

${ }^{b}$ Units (MJy Str $\left.{ }^{-1}\right) /\left(10^{20} \mathrm{~cm}^{-2}\right)$ 
The variation of the $\mathrm{ZE}$ brightness with solar elongation is produced by the fact that lines of sight passing close to the sun sample the inner Solar System, where the dust density is greater. This effect can be used to determine the radial variation of the dust density, but it is sensitive to the optical properties of the interplanetary material. This effect also allows a determination of the infrared emission per unit volume of interplanetary dust at the Earth's orbit, from which the optical properties of the particles may be constrained (Reach 1988).

The variation with observation date is produced by the eccentricity of the Earth's orbit: when at perihelion, the line of sight sampled slightly smaller heliocentric distances. The magnitude of this term gives directly the derivative with respect to heliocentric distance of the infrared emission per unit volume.

We define geocentric azimuth to be the difference between the ecliptic longitude of the line of sight and that of the Sun. At low ecliptic latitudes, the absolute value of this angle is equal to the solar elongation. Here we refer to the odd part of the variation with geocentric azimuth. This term represents an asymmetry of the interplanetary dust distribution which follows the Earth in its orbit around the Sun. Such an effect has never before been observed, but may have been predicted recently. Jackson and Zook (1989) have shown that interplanetary dust particles spiraling from the asteroid belt toward the Sun (due to the Poynting-Robertson effect) can be temporarily trapped in resonances with the Earth. For example, particles in the external 5:4 resonance spend more time trailing the Earth than leading it (cf. their Figure 2).

A very small coefficient for sinusoidal variation of the ZE brightness with ecliptic longitude was found, but the improvement in $\chi^{2}$ was not statistically significant. Such a term would represent either an offset of the cloud center from the Sun or a preferred eccentricity (and argument of perihelion) of particle orbits. Secular gravitational perturbations by the planets (mainly Jupiter) lead to preferred values of the orbital elements as a function of orbital semi-major axis. Lack of a detectable term implies that the actual particle orbits are strongly affected by short time scale fluctuations in the forces felt by the particle-for example, passage through the many resonances in the inner Solar System.

Acknowledgments-This material is based upon work supported by the National Science Foundation under Award No. AST-8818544.

\section{REFERENCES}

Hauser, M.G. 1988, in Comets to Cosmology, ed. A. Lawrence. (Berlin: Springer-Verlag), p. 27. Jackson, A.A. and Zook, H.A. 1989, Nature, 337, 629.

Reach, W.T. and Heiles, C. 1988, in Comets to Cosmology, ed. A. Lawrence. (Berlin: Springer-Verlag), p. 40.

Reach, W.T. 1988, Ap. J., 335, 468. 